\title{
MOLECULES IN STELLAR WINDS
}

Alfred E. Glassgold

Department of Physics, New York University

2 Washington Place, New York, NY 10003, USA

\begin{abstract}
High mass-loss molecular winds are characterstic of two important phases of stellar evolution, very young protostars and stars on the asymptotic branch. Novel chemical processes in these winds suggest a variety of new astronomical observations.
\end{abstract}

\section{PHYSICAL CONDITIONS IN STELLAR WINDS}

Molecules occur in stellar winds if the physical conditions are right: the density must be high and the temperature low. It also helps if the wind can shield itself from the stellar radiation. High density winds are generated by stars at both the beginning and the end of a star's life, i.e. by protostars and evolved red giants. The rate and the duration of the mass loss of these objects are such that a significant fraction of a solar mass (or more) is ejected, large enough to affect the evolution of the stars. How these stars generate their winds is an important, open problem in astrophysics.

Table 1 gives the physical conditions for two, nearby, representative outflows. IRC +10216 is a very bright, evolved (AGB) carbon star with a rich circumstellar chemistry characterized by the formation of complex hydrocarbon and small refractory molecules. The physical conditions in the photochemical region of IRC +10216 are similar to the photodissociation transition region of a dense interstellar cloud. SVS 13 is the prototype of the extremely high velocity (EHV) outflows recently discovered in highly embedded (and therefore very young) protostellar sources. The EHVs are believed to power the moderate velocity, bipolar molecular outflows that are ubiquitous near young stellar objects (Lada 1985). The conditions in the inner regions of a fast, protostellar wind are extreme by the standards of intersteilar chemistry, and define a new branch of astrochemistry. In addition to the short dynamical timescale (about one day), the photorates in the inner wind are extremely large, as illustrated by the photionization rate for neutral carbon atom. However the wind also has a tremendous capacity for self- and mutual-shielding, as witnessed by the maximum value of the far UV optical depth of neutral carbon. 


\begin{tabular}{lll}
\hline \multicolumn{3}{c}{ Table 1. Physical Conditions for Wind Chemistry } \\
Quantity & IRC +10216 & SVS 13 \\
\hline$R_{*}(\mathrm{~cm})$ & $10^{14}$ & $7 \times 10^{11}$ \\
$T_{*}(\mathrm{~K})$ & 2200 & 5000 \\
& & \\
$r(\mathrm{~cm})^{1}$ & $5 \times 10^{16}$ & $10^{12}$ \\
$u_{W}\left(\mathrm{~km} \mathrm{~s}^{-1}\right)^{2}$ & 15 & 150 \\
$\tau_{d y}(\mathrm{~s})$ & $3.3 \times 10^{10}$ & $6.7 \times 10^{4}$ \\
$\dot{M}\left(M_{\odot} \mathrm{yr} \mathrm{s}^{-1}\right)$ & $3 \times 10^{-5}$ & $3 \times 10^{-6}$ \\
$n\left(\mathrm{~cm}^{-3}\right)$ & $2.5 \times 10^{4}$ & $10^{15}$ \\
$T(\mathrm{~K})$ & $\sim 20$ & $\sim 2000$ \\
$G_{o}(\mathrm{C})\left(\mathrm{s}^{-1}\right)^{3}$ & $3 \times 10^{-10}$ & $5 \times 10^{-4}$ \\
$\tau(\mathrm{C})^{4}$ & 10 & $8 \times 10^{6}$ \\
\hline
\end{tabular}

1. Location of intense, in situ chemical activity.

2. Terminal speed.

3. Unshielded photoionization rate of neutral carbon.

4. UV optical depth, assuming all carbon is in CI.

\section{PROTOSTELLAR WINDS}

It is important to distinguish between phenomena associated with the protostellar wind itself and those produced by interaction with the environment, which we refer to as primary and secondary (without any value judgement about their importance). In the former category are the formation and destruction of molecules close to the source of the wind; in the latter are chemical changes induced by the interaction of the wind with infalling, accreting, or ambient gas. One should also distinguish between regions of low- and high-mass star formation. A paradigm for the formation of single, low-mass stars has been developed by Shu and his collaborators (e.g., Shu et al. 1987, 1988), and we focus here on the chemical properties of their primary winds. The Manchester group has discussed some of the chemical phenomena associated with wind interactions (Hartquist et al. 1986, Charnley et al. 1988a, 1988b, 1990).

Typical parameters for a very young protostar are given in Table 1. At a later stage of protostellar evolution, the object becomes visible as a T-Tauri star and the mass-loss rate can be 100 or 1000 times smaller. This case was analyzed by Rawlings, Williams, and Cantó (1988), who found that molecule formation does not occur in such low-density winds. Stimulated by the discovery by Lizano et al. (1988) of both EHV HI and CO, my colleagues and I independently pointed out that the efficiency of molecular synthesis was a sensitive function of density and that $\mathrm{CO}$ was likely to be present in the primary wind of SVS 13 (Glassgold et al. 1989). The simultaneous presence of atomic and molecular gas can be understood in terms of the difficulty in forming and preserving $\mathrm{H}_{2}$ in comparison with $\mathrm{CO}$. 
We recently completed a much more detailed study of a quasi-spherical wind (Glassgold, Mamon, \& Huggins 1991, henceforth GMH), inspired by the X-celerator model of Shu et al. (1988). In order to capture the essential elements of the model, Ruden, Glassgold, and Shu (1989, henceforth RGS) approximated the velocity $u$ and density $n$ by

$$
u(r)=\mathcal{U}(r) v_{W}, \quad n(r)=\frac{C}{r^{2}} \frac{1}{\mathcal{U} \mathcal{A}},
$$

where $\mathcal{U}$ and $\mathcal{A}$ describe the acceleration and collimation of the wind and $C=$ $\dot{M} / 4 \pi m u_{W}$; for SVS 13 as described in Table $1, C=6 \times 10^{35} \mathrm{~cm}^{-1}$. RGS used the simple form, $\mathcal{U}=\mathcal{A}=1-R^{*} / r$ and, after considering wide range of heating and cooling mechanisms, and concluded that:

1. The dominant cooling process is adiabatic expansion.

2. The dominant heating processes are

a. three-body formation of $\mathrm{H}_{2}$ (in the inner envelope)

b. ambipolar diffusion heating (in the outer envelope).

As a result, protostellar winds start out warm but quickly cool down. Note that the inverse process to three-body formation cools the gas and helps limit the maximum temperature. Because ambipolar diffusion heating depends on the electron fraction, both heating mechanisms couple strongly to the chemistry.

To understand protostellar wind chemistry, we start with the results of dynamical and thermal modeling, i.e., both $n$ and $T$ decrease rapidly with distance beyond several protostellar radii. The wind can become molecular by a two-stage process whose details depend sensitively on the density, temperature, and the ultraviolet radiation field $(\mathrm{GMH})$ :

1. Formation of $\mathrm{H}_{2}$

a. Radiative attachment

$$
\begin{aligned}
e+\mathrm{H} \rightarrow \mathrm{H}^{-}+h \nu, & \mathrm{H}^{-}+\mathrm{H} \rightarrow \mathrm{H}_{2}+e \\
\mathrm{H}^{+}+\mathrm{H} \rightarrow \mathrm{H}_{2}^{+}+h \nu, & \mathrm{H}_{2}^{+}+\mathrm{H} \rightarrow \mathrm{H}_{2}+\mathrm{H}^{+}
\end{aligned}
$$

b. Three-body formation

$$
\mathrm{H}+\mathrm{H}+\mathrm{H} \rightarrow \mathrm{H}_{2}+\mathrm{H}, \quad \mathrm{H}+\mathrm{H}_{2}+\mathrm{H} \rightarrow \mathrm{H}_{2}+\mathrm{H}_{2}
$$

The weak radiative attachment reactions are effective for low mass-loss rates and three-body formation at high densities.

2. Hydride \& Heavy Molecule Formation

$$
\mathrm{A}+\mathrm{H}_{2} \rightarrow \mathrm{AH}+\mathrm{H}, \quad \mathrm{AH}+\mathrm{B} \rightarrow \mathrm{AB}+\mathrm{H}
$$

The most important channel for hydride formation involves $\mathrm{A}=\mathrm{O}$ because of the high abundance of $\mathrm{O}$, the low reaction threshold, and large rate coefficient. 
The GMH calculations show that the acceleration and collimation factors considerably enhance molecular synthesis. The density and the dynamical timescale close to the protostar are about 400 and 20 times larger than for the simple case where $\mathcal{U}=\mathcal{A}=1$ (spherically symmetric, impulsively started wind). GMH also find that, except for $\mathrm{H}_{2}$, molecular synthesis is almost complete in that all of the available carbon and silicon are in $\mathrm{CO}$ and $\mathrm{SiO}$ and almost all of the residual oxygen is in $\mathrm{H}_{2} \mathrm{O}$. Even the abundance of molecular hydrogen is quite large, $\sim 0.15$. A novel aspect of the chemistry is $\mathrm{CO} / \mathrm{C}_{t o t} \gg \mathrm{H}_{2} / \mathrm{H}_{\text {tot }}$. Thus, there is generally a substantial abundance of atomic $\mathrm{H}$ capable of emitting the $21 \mathrm{~cm}$ line.

\section{THE PROTOTYPICAL C-RICH AGB STAR IRC +10216}

The circumstellar envelopes of evolved stars are important for both stellar evolution and the interstellar medium. Understanding how evolved stars lose mass means solving the twin problems of the dynamical origins of the winds and the formation of dust. The latter is surely one of the most fundamental problems in astrochemistry, and is likely to involve the close coupling of dynamical, thermal, chemical, and radiative transfer effects. The nearby carbon star IRC +10216 is the prime target for both observational and theoretical studies of circumstellar chemistry and here we focus on the outer envelope. A broader review of circumstellar chemistry has recently been published by Omont (1991). Over forty molecules have been detected in IRC +10216 , mainly in emission at $\mathrm{mm}$ wavelengths, but thirteen have been detected with near infrared absorption spectroscopy. The latter technique is indispensible for detecting symmetric molecules; it also provides important spatial information from analyses of the measured rotational excitation and the line profiles (Keady \& Ridgway 1991).

The molecules observed in the circumstellar envelope of IRC +10216 are formed mainly at three locations:

1. The upper atmosphere of the star $\left(1-2 R_{*}\right)$, characterized by thermal equilibrium and freeze-out of well-bound molecules.

2. The "transition" region $\left(2-30 R_{*}\right)$, where dust is formed and surface reactions occur.

3. The "outer" envelope (beyond $30 R_{*}$ ), which is dominated by photochemistry. These regions may not be all that distinct; they have been introduced to emphasize that different types of chemistry occur in the flow.

Because thermal equilibrium should hold near the photosphere, it might seem reasonable to suppose that the situation there is straightforward. However, the freezeout depends on the run of density and temperature in the upper atmosphere, where the conditions for cool stars with unstable atmospheres (i.e., with strong winds) are not all that well known. Still, careful use of the equilibrium abundance calculations can guide the choice of initial chemical abundances to use for the transition region.

The transition region has long been regarded as nearly intractable because of its complicated and unknown dynamics and poorly understood dust-formation chemistry. 
Among the dynamical effects that have to be considered are the inhomogeneities in the upper atmosphere, shocks produced by stellar pulsations, and the wind generation process itself. The chemistry involves non-equilibrium, inhomogeneous, nucleation and simultaneous treatment of gas-phase and surface chemistry. Omont (1991) gives a critical review of the problems facing the the theory, which he emphasizes must account for the partial condensation of the wind. Despite the formidable difficulties, there seems to be a consensus that a chemical-kinetic approach can provide some insights into the precursors of the solid phases (e.g., Frenklach \& Feigelson 1989).

Equally important are observations that probe the region of dust formation. For example, Danchi et al. (1989) have made new $10 \mu \mathrm{m}$ interferometric measurements of IRC +10216 which indicate that dust forms close to the star, $\sim 0.05^{\prime \prime}$ or within $2-3 R_{*}$, and most efficiently at minimum luminosity. The dust continues to evolve physically and chemically as it moves into the outer envelope. Evidence for depletion of the gas onto dust is provided by interferometric measurements of the mm lines of $\mathrm{SiS}$ (Bieging \& Rieu 1989), which peak strongly right on IRC +10216 and then decrease rapidly in a few arcseconds $\left(10^{15}-10^{16} \mathrm{~cm}\right)$. Evidence for molecule formation in this same region has been deduced from the lineshapes of several molecules in the $10 \mu \mathrm{m}$ window (Keady \& Ridgway 1991). In particular, $\mathrm{NH}_{3}, \mathrm{CH}_{4}$, and $\mathrm{SiH}_{4}$ all appear to form in the region from $10-30 \mathrm{R}_{*}$ (or $1-3 \times 10^{15} \mathrm{~cm}$ ). This is in accord with thermal equilibrium calculations for the upper atmosphere of the star, which give very low abundances for these fully hydrogenated species. The presumption is that they are formed on grains.

The photochemical chains introduced by Huggins \& Glassgold (1982) form the basis of the theory of the outer envelope. Two of the earliest photochains considered start with $\mathrm{CO}$ and $\mathrm{C}_{2} \mathrm{H}_{2}$ and lead to chemically reactive ions and radicals. The theory predicts variations on a spatial scale that can now be measured with existing facilities like the IRAM 30-m and the Nobeyama 45-m telescopes and several mm arrays. Pioneering work by Bieging \& Rieu $(1988,1989)$ demonstrate the technique and confirm the qualitative predictions of the photochemical model of the outer envelope. The following general comments on the connections between the theory and observation are relevant in this connection.

1. The molecules in the outer envelope are either (a) progenitors or (b) synthesis products. A progenitor may have survived the transition region intact (e.g., $\mathrm{CO}$ ), particpated in dust fromation (e.g., $\mathrm{C}_{2} \mathrm{H}_{2}$ ), or have been synthesized in the transition region (e.g., $\mathrm{NH}_{3}, \mathrm{CH}_{4}$, and $\mathrm{SiH}_{4}$ ). Progenitors and synthesis products have essentially different spatial distributions, respectively, (a) uniform, with a cut-off and (b) shell. Interferometric measurements with angular resolution better than $10^{\prime \prime}$ can distinguish between them. This is obviously important in the absence of a theory of the inner regions.

2. Photodissociation by the interstellar radiation field eventually destroys all 
simple molecules, leaving all atoms except $\mathrm{H}$ and $\mathrm{O}$ singly ionized. The finite size of a molecular distribution is determined by the unshielded photorate and by shielding. In addition to $\mathrm{CO}$ (discussed above), measurements of molecular cutoffs in IRC +10216 have been published for $\mathrm{HCN}$ (Bieging, Chapman, \& Welch 1984), $\mathrm{C}_{2} \mathrm{H}, \mathrm{HNC}, \mathrm{HC}_{3} \mathrm{~N}$ (Bieging \& Rieu 1988), and SiS (Bieging \& Rieu 1989). The far UV dust extinction needed to fit the observations is greater than that of interstellar dust, consistent with a smaller size for newly formed grains (Huggins, Morris, \& Glassgold 1984, TruongBach et al. 1987). In principle, measurements of the decline in abundances at large distances for a well selected set molecules with known photodissociation rates could determine the far UV optical properties of the circumstellar dust of IRC +10216 .

A new direction for circumstellar chemistry involves silicon: seven silicon molecules have already been identified in IRC $+10216\left(\mathrm{SiH}_{4} \mathrm{SiC}, \mathrm{SiC}_{2}, \mathrm{SiC}_{4}, \mathrm{SiO}, \mathrm{SiN}\right.$, and $\left.\mathrm{SiS}\right)$. Glassgold, Lucas, \& Omont (1987) first proposed that $\mathrm{SiC}_{2}$ was synthesized by the condensation reactions

$$
\begin{aligned}
& \mathrm{Si}^{+}+\mathrm{C}_{2} \mathrm{H}_{2} \rightarrow \mathrm{SiC}_{2} \mathrm{H}^{+}+\mathrm{H} \\
& \mathrm{Si}^{+}+\mathrm{C}_{2} \mathrm{H} \rightarrow \mathrm{SiC}_{2}^{+}+\mathrm{H}, \quad \mathrm{SiC}_{2}^{+}+\mathrm{H}_{2} \rightarrow \mathrm{SiC}_{2} \mathrm{H}^{+}+\mathrm{H},
\end{aligned}
$$

on the assumption that $\mathrm{Si}^{+}$comes from photoionization of $\mathrm{Si}$; they later suggested that the $\mathrm{Si}$ comes from the photodissociation of SiS (observed in the outer envelope with an abundance of $\sim 3 \times 10^{-7}$, e.g., Bieging \& Rieu 1989) and proposed that ion-molecule reactions of $\mathrm{SiS}$ could produce the entire silicon carbide family. The products of the SiS chain display the customary shell distribution (Glassgold \& Mamon 1990), with an unusually broad $\mathrm{SiC}_{2}$ distribution. Howe \& Millar (1990) proposed a similar model but assumed that $\mathrm{SiC}_{2}$ was a progenitor molecule with an abundance of $7.5 \times 10^{-8}$. Several ongoing projects to map $\mathrm{SiC}_{2}$ in IRC +10216 , e.g., with the IRAM $30 \mathrm{~m}$ telescope and interferometer (Lucas 1991) and the BIMA array (Gensheimer et al. 1991), show that $\mathrm{SiC}_{2}$ is distributed in a shell, in qualitative agreement with the theory of Glassgold \& Mamon (1990).

The recent detection of SiN (Turner 1991) links circumstellar silicon and nitrogen chemistry. Turner suggests that is formed by

$$
\mathrm{Si}^{+}+\mathrm{NH}_{3} \rightarrow \mathrm{HSiNH}^{+}+\mathrm{H} .
$$

This route should work (if the recombination of $\mathrm{HSiNH}^{+}$produces $\mathrm{SiN}$ ) because $\mathrm{NH}_{3}$ is observed in the IR at the beginning of the outer envelope with an abundance $\sim 7.5 \times 10^{-8}$ (Keady \& Ridgway 1991). Turner also suggests that the $\mathrm{Si}^{+}$comes from the silane photochain, again based on the fact that $\mathrm{SiH}_{4}$ is observed in the dust formation region with an abundance of $\sim 10^{-7}$. However, silane is just one of several sources of $\mathrm{Si}$ and $\mathrm{Si}^{+}: \mathrm{SiO}\left(x \sim 4 \times 10^{-7}\right)$, $\mathrm{SiS}\left(x \sim 3 \times 10^{-7}\right), \mathrm{SiH}_{4}\left(x \sim 10^{-7}\right)$, and possibly $\mathrm{SiC}_{2}$ and $\mathrm{Si}_{2} \mathrm{C}$. It is interesting that, assuming that its photospheric abundance of is solar, more than $2 \%$ of the silicon survives the dust-formation process in IRC +10216 . 


\section{CONCLUSION}

The above discussion of circumstellar chemistry illustrates the close links between dynamical, thermal, chemical, and radiative transfer effects. A similar coupling occurs in interstellar clouds. The advantage of circumstellar chemistry is that realistic models of the flow can be obtained so that basic thermal and chemical processes can be tested in well-defined environments.

\section{REFERENCES}

Bieging, J., Chapman, B., \& Welch, W.J. 1984, ApJ, 285, 256

Bieging, J. \& Rieu, N.-Q., 1988, ApJ (Letters), 329, L107

Bieging, J. \& Rieu, N.-Q., 1989, ApJ (Letters), 343, L25

Charnley, S.B., Dyson, J.E., Hartquist, T.W., Williams., D.A., 1988a, MNRAS,

231,$169 ; 1988 \mathrm{~b}$, MNRAS, 235, 1257; 1990, MNRAS, 243, 405

Danchi, W.C., Bester, M., Degiacomi, C.G. McCullough, P.R., Townes, C.H. 1990, ApJ (Letters), 359, L59

Frenklach, M. \& Feigelson, E.D. 1989, ApJ 341, 372

Gensheimer, P., Likkel, L., Snyder, L.E., 1991, BAAS, 23, 911

Glassgold, A.E., Lucas, R., Omont, A. 1986, A\&A, 157, 35

Glassgold, A.E. \& Mamon, G.A., 1990, Chemistry and Spectroscopy of Interstellar

Molecules, ed. N. Kaifu, (Tokyo), in press

Glassgold, A.E., Mamon, G.A., Huggins, P.J., 1989, ApJ (Letters). 336, L29

Glassgold, A.E., Mamon, G.A., Huggins, P.J., 1991, ApJ 373, 254 (GMH)

Hartquist, T.W., Dyson, J.E., Pettini, M., Smith, L.J., 1986, MNRAS 221, 715

Howe, D.A. \& Millar, T.J., 1990, MNRAS, 244, 444

Huggins, P.J. \& Glassgold, A.E., 1982, ApJ, 252, 201

Huggins, P.J., Glassgold, A.E., \& Morris, M., 1984 ApJ, 279, 284

Keady, J.J. \& Ridgway, S., 1991, preprint

Lizano, S., Heiles, C., Rodriguez, L.F., Koo, B.-C., Hasegawa. T., Hayashi, S.,

Mirabel, I.F., 1988, ApJ, 328, 763

Lucas, R. 1991, Astrochemistry of Cosmic Phenomena, in press

Nejad, L.A.M., Millar, T.J., Freeman, A., 1984, MNRAS, 134, 129

Omont, A. 1991, Chemistry in Space, eds. J.M. Greenberg \& V. Pirrinello,

(Kluwer:Netherlands), p.171

Rawlings, J.M.C., Williams, D.A., Cánto, J., 1988, MNRAS, 230, 695

Ruden, S.P., Glassgold, A.E., Shu, F.H., 1990, ApJ, 361, 546 (RGS)

Shu, F.H., Adams, F.C., Lizano, S., 1987, ARAA, 25, 23

Shu, F.H., Lizano, S., Ruden, S.P., Najita, J., 1988, ApJ (Letters), 328, L19

Truong-Bach, Rieu, N.-Q., Omont, A., Oloffson, H., Johansson, L.E.B. 1987,

A\&A 176, 285

Turner, B.E., 1991, BAAS, 23, 933 


\section{QUESTIONS AND ANSWERS}

L.d'Hendecourt: Apparently, $2 \%$ of the silicon survives dust formation. Is this a measured value? How does this compares with the strong depletion of $\mathrm{Si}$ in the diffuse ISM?

A.E.Glassgold: Infrared measurements of IRC+10216, summarized by Keady \& Redgway in the 1991 Ap.J. are the basis of the approximate $2 \%$ figure. In the diffuse interstellar medium, it is about the same, but silicon is much more depleted in dense regions.

D.A. Williams: There is a remark on the requiremente for dust with high FUV extinction in IRC+10216. I think this indeed likely. There is no reason to expect that dust in IRC+10216 should have the same extintion character as the mean interstellar dust. In IRC+10216 dust is likely to be carbonaceous and hydrogen-rich. Films made in the laboratory of hydrogen-rich amorphous carbon have a high band gap and will automatically provide high far UV extinction. Such grains, if they survive to the ISM, will lose hydrogen and become more typical of general ISM, with stronger visible extinction, at the expense of the far UV extinction.

\section{A.E.Glassgold: Neat!}

J.M.C.Rawlings: (1) What is the source of your radiation field? A black-body at T 2000 - $5000 \mathrm{~K}$ (a 2000 $\mathrm{K}$ black-body is fainter in the UV than the I-S radiation field) surely does not emit a significant amount of UV photons bearing in mind the high densities in the outflow. Is Ly $\alpha$ trapping included in the model? (2) In the three cases for the temperature profiles that you presented, you included $a\left(1-R_{*} / r\right)$ factor. What is the justification for this?

A.E.Glassgold: (1) The protostellar radiation field was usually represented ed by a blackbody spectrum of $5000 \mathrm{~K}$.Depending on the spectral properties of the absorber, the photorates near the protostar are typically 6-10 orders of magni- tude larger than the interstellar medium. We also investigated protostellar spectra ctra with large UV excesses and found that the abundance of $\mathrm{CO}$ is quite robust. We have never considered using a photospheric temperature as small as $2000 \mathrm{~K}$ for a low mass protostar.It is true that the proper measure of the effects of the radiation field is the parameter $G / n$, where $G$ is a photorate and $n$ is the density.In our variational studies, this parameter well exceeded the value for the diffuse interstellar medium. Lyman alpha trapping was included in the ionization theory of the $\mathrm{H}$ atom.

(2) The modulation factors for velocity and collimation were chosen arbitrarily to go to unity in a few stellar radii, as suggested by the dynamical wind model of Shu et al.(1988). We have since verified that the choice made by RGS gives a good representation of the flow obtained by exact integration of of the appropriate MHD equations.

J.P.Maillard: If I understand the reaction proposed to produce SiN even if it is very unabundant from thermo-equilibrium reactions in carbon-rich environment, it supposes that it forms at the edge of the circumstellar envelope. But it is where it should be destroyed by photoionization. Are the models able to reconcile this contradiction?

A.E.Glasssgold: SiN should have a "shell" distribution due to the combined effects of production by $\mathrm{Si}^{+}$ and radiative destruction. There is little $\mathrm{SiN}$ in the inner envelope because the abundance of $\mathrm{Si}^{+}$goes to zero, and photodissociation limits the ammount of SiN in the outer envelope. 